\title{
Exercise-Induced Changes in Branched Chain Amino Acid/Aromatic Amino Acid Ratio in the Rat Brain and Plasma
}

\author{
Koji OKAMURA, Futoshi MATSUBARA, Yasuyuki YOSHIOKA, \\ Noriaki KIKUCHI, Yuko KIKUCHI and Hideaki KOHRI \\ Otsuka Pharmaceutical Co.. Ltd., Saga Research Institute. 5006-5 Aza Higashiyama. \\ Ohaza Ohmagari, Higashi Sefuri-mura, Kanzaki-gun, Saga 842-01, Japan
}

Accepted July 4, 1987

\begin{abstract}
Central fatigue was induced by running rats on a treadmill. Amino acid and ammonia metabolism in the brain and blood were followed with time to correlate its changes with physical exhaustion. The blood ammonia level did not change during running, but was prominently increased at exhaustion. The brain level of ammonia was also prominently high at the end of exercise with a time course of change similar to that of the blood level. Plasma concentrations of branched chain amino acids (BCAA) and aromatic amino acids (AAA) increased as the animals continued to run: however, the plasma BCAA/AAA ratio was definitely depressed at exhaustion. This was also true with the brain BCAA/AAA ratio. A positive correlation was demonstrated between the plasma and brain BCAA/AAA ratios at $r=0.5040$ and $P<0.05$. These exercise-related changes suggest that physical exercise-induced central fatigue involves not only an increase in brain ammonia, but also a disturbance in brain amine metabolism accompanying plasma and brain BCAA/AAA ratio depression. Furthermore, the ammonia level and BCAA/ $A A A$ ratio in the brain correlated with those in the blood. It is reasonable to consider that the blood ammonia concentration and plasma BCAA/AAA ratio may serve as important indices of the clinical condition of exercise-induced central fatigue.
\end{abstract}

Carbohydrates and fats are the principal sources of energy during physical exercise, but protein in the form of amino acids is also mobilized for energy production to a certain degree (1). The types of amino acids utilized in contracting skeletal muscle are branched chain amino acids (BCAA) including valine, isoleucine and leucine (1). This is supported by reports that the plasma concentration of BCAA decreased under sustained exercise (2-7).

BCAA compete with aromatic amino acids (AAA) such as tyrosine and phenylalanine, which are precursors of norepinephrine (NE) and dopamine (DA), at the blood brain barrier (BBB) for uptake into the brain (810). Fischer and other workers hypothesized that a fall in the plasma BCAA/AAA ratio leads to an increase of brain AAA, causing an increase of false neurotransmitters as represented by phenylethanolamine and oc- topamine and a decrease of NE and DA as probable factors affecting the central nervous system (CNS) (10-14). When physical exercise is so intense as to alter the blood $B C A A$ concentration and thereby the BCAA/ AAA ratio, brain amine metabolism may be impaired, thus inducing impairment of CNS function. It may be reasonable that this impairment of CNS function would correlate to an exercise-induced subjective sensation of fatigue; i.e., central fatigue.

It is common knowledge that blood ammonia accumulates during exercise (4, 15. 16). Since excess ammonia elicits a toxic effect on the CNS (17-19), exercise-induced brain ammonia accumulation may also contribute to the induction of central fatigue.

In the present study using rats, we examined the time courses of brain and blood concentrations of BCAA. AAA and ammonia during treadmill exercise loading with an aim 
to correlate these changes to the induction of central fatigue.

\section{Materials and Methods}

Experimental procedure: Male SpragueDawley rats (Charles River Japan, Inc.) at 5 weeks of age were acclimatized for 7 days prior to the running test under an artificial reversed cycle of $12 \mathrm{hr}$ of dark during the day (7:00-19:00) and $12 \mathrm{hr}$ of light during the night (19:00-7:00) in an animal room maintained at $23 \pm 0.5^{\circ} \mathrm{C}$ with a relative humidity of $55 \pm 5 \%$. Food (MF. Oriental Co., $L t d$.) and water were provided freely.

The animals were then familiarized with a rodent treadmill (NOK-10. Nippon Cage Co., Ltd.) for 3 days at daily increasing work intensities of $20 \mathrm{~m} / \mathrm{min}$ on the initial day, 30 $\mathrm{m} / \mathrm{min}$ on the second day and $35 \mathrm{~m} / \mathrm{min}$ on the last day for $20 \mathrm{~min}$ each day.

The animals were fasted for $15 \mathrm{hr}$ from 18:00 on the previous day and subjected to running on the treadmill at the rate of $35 \mathrm{~m} /$ min until exhaustion. The experiment was commenced at 9:00 in view of the nocturnal behavior of rats. The animals were considered to be exhausted when they stopped running and were unable to stand after being laid flat.

Rats were sacrificed after 0,15,30,60 and 90 min of exercise, upon exhaustion, and 15. 30 and $60 \mathrm{~min}$ after exercise. The blood and whole brain were sampled immediately following sacrifice. The whole brain was frozen in liquid nitrogen. The blood was centrifuged at 3000 r.p.m. at $4{ }^{\circ} \mathrm{C}$, and the plasma was collected. The concentrations of free amino acids in the whole brain and plasma were determined (20) using a Hitachi high speed amino acid analyzer (Model 835$50)$. The concentrations of ammonia in the brain and blood were determined according to the method of Okuda and Fujii (21). with some modifications.

Statistics: Data are presented as the means $\pm S$.E. and were analyzed by Student's $t$-test, with $P$ values of less than $5 \%$ for statistical significance.

\section{Results}

1. Blood and brain ammonia concentrations: Blood ammonia gradually increased from a pre-exercise level of $121 \pm 17 \mu \mathrm{g} / \mathrm{dl}$ as

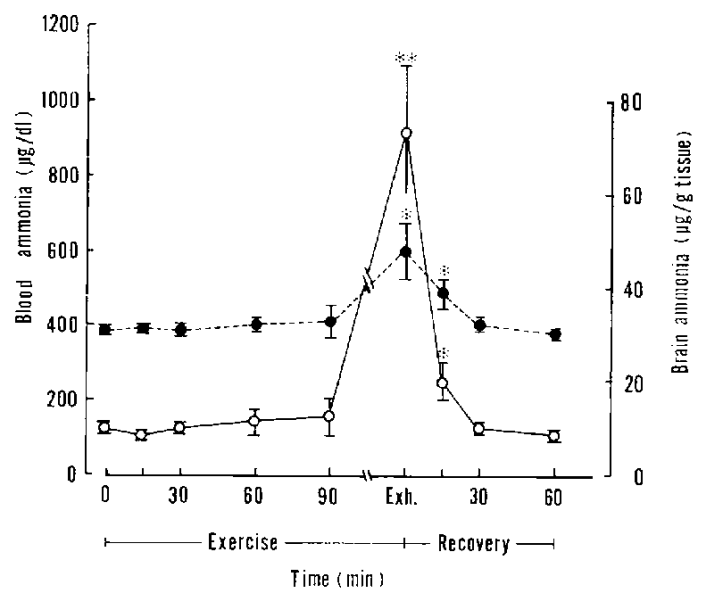

Fig. 1. Blood and brain ammonia concentrations during exercise and recovery periods. Rats were run on a rodent treadmill at $35 \mathrm{~m} / \mathrm{min}$ and sacrificed at the times indicated in the figure. Each point represents the mean $\pm S$.E. of 6 to 7 animals. ${ }^{*} P<0.05$ and ${ }^{*} P<0.01$, compared to the pre-exercise value. Exh.: exhaustion. $O-O$ : blood ammonia and - brain ammonia.

running continued to reach a 7.5 -fold higher level of $913 \pm 182 \mu \mathrm{g} / \mathrm{dl}$ at exhaustion $(P<0.01)$. This level fell back down to the pre-exercise level after $30 \mathrm{~min}$ of recovery (Fig. 1).

The brain ammonia level followed a course similar to that of the blood level. The preexercise level of $30.4 \pm 1.0 \mu \mathrm{g} / \mathrm{dl}$ increased to $47.8 \pm 5.9 \mu \mathrm{g} / \mathrm{dl}$ at exhaustion $(P<0.05)$ and returned to the pre-exercise level after $30 \mathrm{~min}$ of recovery (Fig. 1).

The mean duration of running time of rats was $113.1 \pm 4.5 \mathrm{~min}$.

2. Plasma and brain free BCAA and AAA concentrations: Free BCAA and AAA in the plasma showed a work load-related increase during exercise, reaching a peak at exhaustion. The BCAA/AAA ratio gradually became depressed since AAA increased more than BCAA at each measurement. The ratios of $2.992 \pm 0.100$ at the start and $2.506 \pm 0.074$ at exhaustion were significantly different from each other $(P<0.01)$ (Fig. 2$)$.

Free BCAA and AAA in the brain showed the greatest increase after $60 \mathrm{~min}$ of exercise. followed by a gradual decrease. However, the concentrations remained moderately high at 

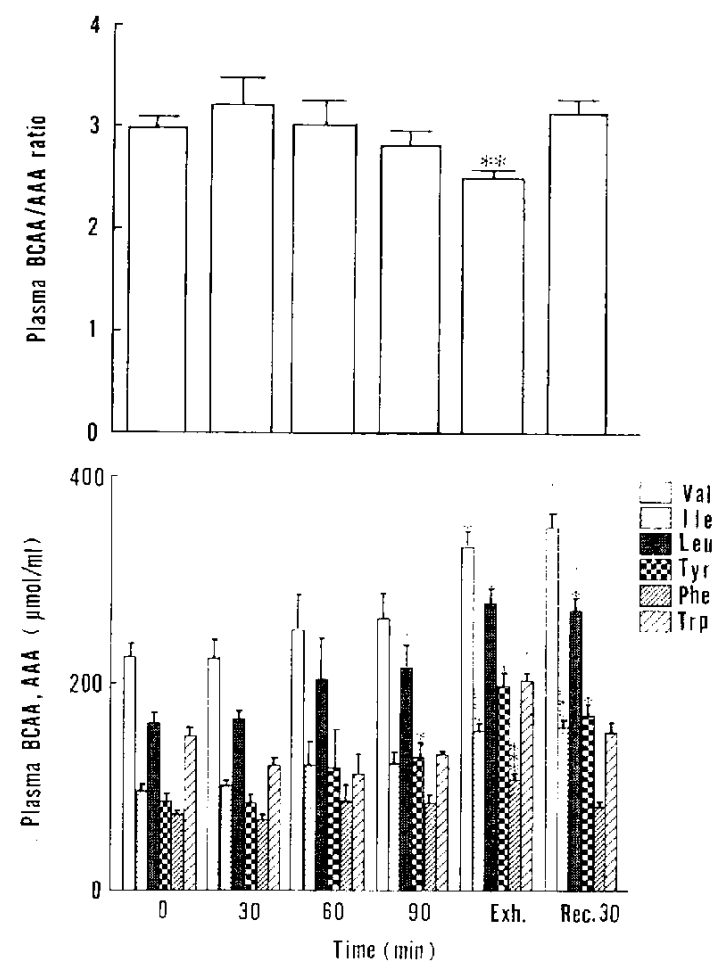

Fig. 2. Plasma BCAA/AAA molar concentration ratio (top) and BCAA and AAA concentrations during exercise and recovery periods. See details in the Materials and Methods section of the text. Each point represents the mean $\pm S$.E. of 5 to 6 animals. ${ }^{*} P<0.05,{ }^{* *} P<0.01$ and ${ }^{* *} P<0.001$, compared to the pre-exercise value. Val: valine, Ile: isoleucine, Leu: leucine. Tyr: tyrosine. Phe: phenylalanine. Trp: tryptophan, Exh.: exhaustion and Rec.: recovery.

exhaustion. The BCAA/AAA ratio appeared to reflect the plasma ratio: the mean ratio of $2.562 \pm 0.045$ at the start dropped significantly to $2.111 \pm 0.089$ at exhaustion $(P<0.01$ ) (Fig. 3).

The plasma and brain BCAA/AAA ratios showed a significant positive correlation $(r=0.5040, P<0.05)$ (Fig. 4).

The decrease in the plasma and brain $B C A A / A A A$ ratios as well as the increase in the blood and brain ammonia concentrations was considered to be indicative of the occurrence of central fatigue.

\section{Discussion}

Physical exercise or movement accelerates production of ammonia in the muscle purine-
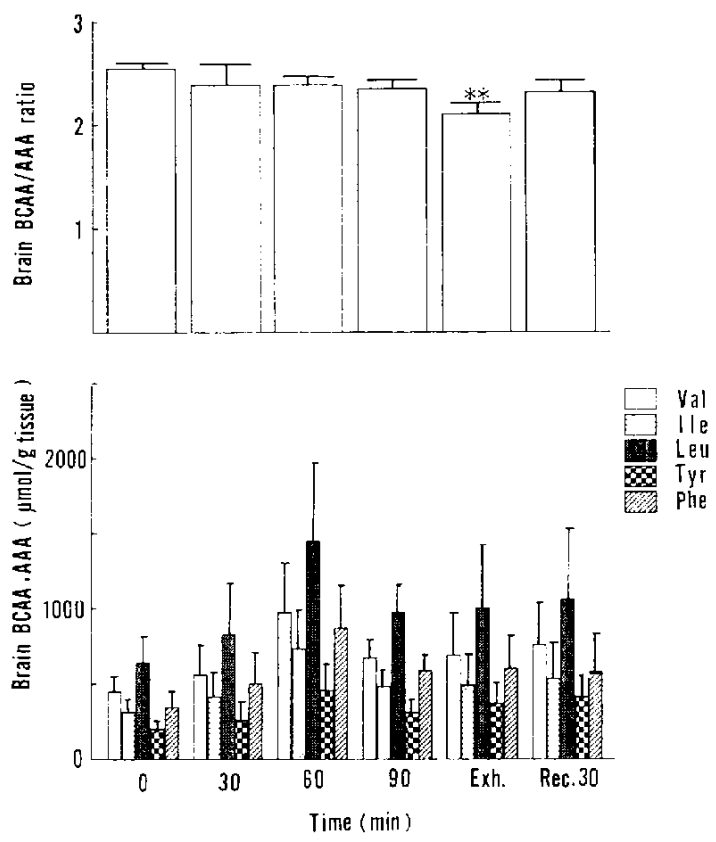

Fig. 3. Brain BCAA/AAA molar concentration ratio (top) and BCAA and AAA concentrations during exercise and recovery periods. See details in the Materials and Methods section of the text. Each point represents the mean $\pm S$.E. of 5 to 6 animals. ${ }^{*} P<0.01$ compared to the pre-exercise value. Val: valine, lle: isoleucine, Leu: leucine, Tyr: tyrosine, Phe: phenylalanine, Exh.: exhaustion and Rec.: recovery.

nucleotide cycle (22), resulting in its increased blood concentration (4, 15, 16). Circulating ammonia is taken up by the liver and is mostly detoxified in the hepatic urea cycle; however, accelerated ammonia formation tends to exceed hepatic detoxication due to diminishing hepatic blood flow during exercise (23). The present study in the rat revealed that exercise caused an increase in the blood concentration of ammonia, as was previously reported in humans, and also an increase in the brain concentration of ammonia, reaching a statistically significant level at exhaustion following a time course similar to that of the blood concentration. Since ammonia is toxic to the CNS (17-19). its increased brain concentration might be considered a probable factor in central fatigue due to physical exercise.

BCAA, which are utilized in skeletal muscle 


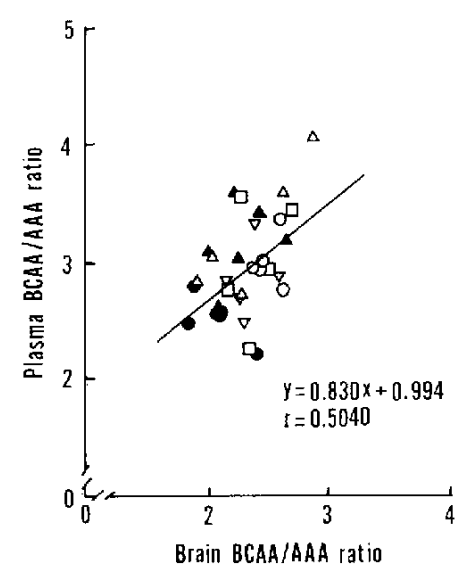

Fig. 4. Correlation between plasma and brain BCAA/AAA molar concentration ratios. See details in the Materials and Methods section of the text. The line represents the linear regression of the two ratios from the values at $0(0), 30(\triangle), 60(\square)$ and $90 \mathrm{~min}$ of exercise ( $\nabla$ ), exhaustion (O) and after 30 min of recovery $(\mathbf{A})$.

during exercise (1), have been reported to decrease in the plasma as exercise continued (2. 3), while they have also been reported to increase during exercise $(6,7)$. On the other hand, plasma AAA were reported to remain constant in one study (5) and to incresse in other studies during exercise (4, 6, 7). In contrast, both free BCAA and AAA increased in the plasma during exercise in the present study. These differences in experimental findings were related to differences in the conditions of exercise loading, since the metabolic efficiency of protein in muscles during exercise is dependent on the intensity and duration of exercise (1).

The plasma BCAA/AAA ratio in the present study gradually dropped to a significantly low level at exhaustion as the rats continued to run. The plasma concentrations of both BCAA and AAA were elevated by exercise. Despite increases in the two types of amino acids. the BCAA/AAA ratio fell. The fall was associated with the increase of AAA over BCAA. BCAA and AAA are known to compete with each other at the BBB for uptake into the brain ( $8-10)$, and so the fall in the plasma BCAA/AAA ratio observed in the present study eventually resulted in an increase of brain AAA. Fischer and other workers cor- related the fall in the plasma $B C A A / A A A$ ratio to an increase of $A A A$, which results in a disturbance of brain amine metabolism as represented by increased amounts of false neurotransmitters and decreased amounts of neurotransmitters, indicating a high likelihood of CNS impairement (10-14). In agreement with these previous findings, the present study also showed a positive correlation between the brain and plasma BCAA/AAA ratios, and the ratios were definitely low at exhaustion of the animals. It may be reasonable to hypothesize that physical exercise-induced central fatigue seems to involve impaired brain amine metabolism secondary to a fali in the plasma and brain BCAA/AAA ratios.

Chaouloff et al. $(24,25)$ recently observed that plasma and brain concentrations of tryptophan (Trp) and serotonin (5-HT) increased with exercise in rats. Acworth et al. (26) also observed an increase of Trp and 5 $\mathrm{HT}$ in the rat brain after exercise and related the increase to the occurrence of central fatigue. The present study did not measure these amino acids and amines in the brain. but it did confirm a significant increase of Trp in the plasma when rats were run to exhaustion. It is probable that the brain Trp concentration was also significantly high at exhaustion.

In conclusion, exhaustive exercise caused a fall in the plasma and brain BCAA/AAA ratios and a simultaneous increase in the blood and brain ammonia concentrations in the present study in rats. If this is true with human exercise, these changes in the blood and brain may serve as important factors contributing to exercise-induced central fatigue in man. Further investigation may suggest possible measures to alleviate central fatigue. It is reasonable to assume that when the plasma and brain BCAA/AAA ratios are returned to normal after being disturbed, brain ammonia would be reduced or removed and brain amine metabolism would eventually return to normal, resulting in alleviation of central fatigue or early recovery of normal health. Fischer et a!. $(27,28)$ proposed a similar means of correcting plasma and brain BCAA/AAA ratios in patients with hepatic encephalopathy. Since the ammonia level 
and BCAA/AAA ratio in the brain correlate with those in the blood, these indices may be used as reliable indices for the occurrence and severity of central fatigue during and after exercise.

\section{References}

1 Lemon, P.W.R. and Nagle, F.J.: Effects of exercise on protein and amino acid metabolism. Med. Sci. Sports Exerc. 13, 141-149 (1981)

2 Bergström, J., Fürst, P. and Hultman, E.: Free amino acids in muscle tissue and plasma during exercise in man. Clin. Physiol. 5, 155-160 (1985)

3 Refsum, H.E., Gjessing, L.R. and Stromme, S.B.: Change in plasma amino acid distribution and urine amino acids excretion during prolonged heavy exercise. Scand. J. Clin. Lab. Invest. 39, 407-413 (1979)

4 Eriksson, L.S., Broberg, S., Bjorkman, O. and Wahren, J.: Ammonia metabolism during exercise in man. Clin. Physiol. 5, 325-336 (1985)

5 Poortmans, J.R., Siest, G., Galteau, M.M. and Houot, O.: Distribution of plasma amino acids in humans during submaximal prolonged exercise. Eur. J. Appl. Physiol. 32, 143-147 (1974)

6 Wahren, J., Felig, P., Hendler, R. and Ahlborg, G.: Glucose and amino acid metabolism during recovery after exercise. J. Appl. Physiol. 34, 838-845 (1973)

7 Dhom, G.L., Beecher, G.R., Warren, R.Q. and Williams, R.T.: influence of exercise on free amino acid concentrations in rat tissues. J. App!. Physiol. 50, 41-44 (1981)

8 Oldendorf, W.H.: Brain uptake of radiolabeled amino acids, amines, and hexoses after arterial injection. Am. J. Physiol. 221, 1629-1639 (1971)

9 Pardridge, W.M.: The role of blood-brain barrier transport of tryptophan and other neutral amino acids in the regulation of substrate-limited pathways of brain amino acid metabolism. J. Neural Transm. [Supp.] 15, 43-54 (1979)

10 James, J.H., Escourrou, J. and Fischer, J.E.: Blood-brain neutral a mino acid transport activity is increased after portacaval anastomosis. Science 200, 1395-1397 (1978)

11 Fischer, J.E. and Baldessarini, R.J.: False neurotransmitters and hepatic failure. Lancet 2, 75-80 (1971)

12 Fischer, J.E., Yoshimura, N., Aguirre, A., James, J.H., Cummings, M.G., Abel, R.M. and Diendoerfer, F.: Plasma amino acids in patients with hepatic encephalopathy: Effects of amino acid infusions. Am. J. Surg. 127, 40-47 (1974)

13 Fischer, J.E., Funovics, J.M., Aguirre, A., James,
J.H., Keane, J.M., Wesdorp, R.I.C., Yoshimura, $\mathrm{N}$. and Westman, T.: The role of plasma amino acids in hepatic encephalopathy. Surgery 78 , 276-290 (1975)

14 Rosen, H.M. Yoshimura, N., Hodgman, J.M. and Fischer, J.E.: Plasma amino acid patterns in hepatic encephalopathy of differing etiology. Gastroenterology 72. 483-487 (1977)

15 Banister, E.W., Allen, M.E., Mekjavic, I.B., Singh, A.K., Legge, B. and Mutch, B.J.C.: The time course of ammonia and lactate accumulation in blood during bicycle exercise. Eur. J. Appl. Physiol. 51, 195-202 (1983)

16 Buono, M.J., Clancy, T.R. and Cook, J.R.: Blood lactate and ammonia ion accumulation during graded exercise in humans. J. Appl. Physiol. 57. 135-139 (1984)

17 James, J.H., Zipard, V., Jeppsson, B. and Fischer, J.E: Hyperammonemia, plasma amino acid imbalance, and blood-brain amino acid transport: A unified theory of portal-systemic encephalopathy. Lancet 2, 772-775 (1979)

18 McCandless, D.W. and Schenker, S.: Effect of acute ammonia intoxication on energy stores in the cerebral reticular activating system. Exp. Brain Res. 44, 325-330 (1981)

19 Schenker, S., McCandless, D.W., Brophy, E. and Lewis, M.S.: Studies on the intracerebral toxicity of ammonia. J. C!in. Invest. 46, 838-848 (1967)

20 Kido, Y., Sugiyama, K., Kashiyama, E., Nakao, S. Kudo, S., Suda, T., Miyamoto, G., Shimizu, T. and Kohri, H.: Effects of SF-1008C, an enteral nutrient for liver failure, on hepatic encephalopathy. Japan. Pharmacol. Ther. 13, 495-506 (1985) (in Japanese)

21 Okuda, H. and Fujii, S.: Direct colorimetric assay of blood ammonia. Medicine Today (SaishinIgaku) 21, 622-627 (1966) (in Japanese)

22 Lowenstein, J.M.: Ammonia production in muscle and other tissues: The purine nucleotide cycle, Physiol. Rev. 52, 382-414 (1972)

23 Waren, J., Felig, P., Ahlborg, G. and Jorfeldt, L.: Glucose metabolism during leg exercise in man. J. Clin. Invest. 50, 2715-2725 (1971)

24 Chaouloff, F., Laude, D., Guezennec, Y. and Elghozi, J.L.: Motor activity increases tryptophan, 5-hydroxyindoleacetic acid, and homovanillic acid in ventricular cerebrospinal fluid of the conscious rat. J. Neurochem. 46, 1313-1316 (1986)

25 Chaouloff, F., Kennett, G.A., Serrurrier, B., Merino, D. and Curzon, G.: Amino acid analysis demonstrates that increased plasma free tryptophan causes the increase of brain tryptophan 
during exercise in the rat. J. Neurochem. 46, 1647-1650 (1986)

26 Acworth, I., Nicholass, J., Morgan, B. and Newsholme, A.: Effect of sustained exercise on concentrations of plasma aromatic and branchedchain amino acids and brain amines. Biochem. Biophys. Res. Commun. 137, 149-153 (1986)

27 Fischer, J.E., Rosen, H.M., Ebeid, A.M., James, J.H., Keane, J.M. and Soeters, P.B.: The effect of normalization of plasma amino acids on hepatic encephalopathy in man. Surgery 80, 77-91 (1976)

28 Freund, H., Dienstag, J., Lehrich, J., Yoshimura, N., Bradford, R.R., Rosen, H., Atamian, S., Slemmer, E., Holroyde, J. and Fischer, J.E.: Infusion of branched-chain enriched amino acid solution in patients with hepatic encephalopathy. Ann. Surg. 196, 209-220 (1982) 